\title{
NONLINEAR ANALYSIS IN GEODESIC METRIC SPACES
}

\author{
IAN SEARSTON
}

(Received 3 June 2015; first published online 5 August 2015)

\section{Mathematics subject classification: primary 53C23; secondary 47H09, 58B99.}

Keywords and phrases: geodesic, nonlinear analysis, convexity, nonconstant curvature, weak convergence, weak sequential convergence, hyperplanes, fixed points, nonexpansive, projections, reflections.

This thesis deals with nonlinear analysis in geodesic metric spaces, particularly in CAT(0) spaces. A major aim is to investigate the convex feasibility problem associated with the nonempty closed convex sets $A$ and $B$. This problem is normally investigated in a Hilbert space setting, but in this work we have placed it in a CAT(0) setting.

Since CAT(0) spaces are geodesic metric spaces, we begin with a summary of preliminaries that will be used in the rest of our work. We begin with metrics and metric spaces and include convergence and completeness because of their importance in later work. We then deal with linear and normed linear spaces and importantly Hilbert spaces and weak convergence therein.

We then introduce geodesic metric spaces. We begin with definitions of arcs, curves and geodesics and then deal with convexity, both Menger and Busemann, including a proof of Menger's theorem. We conclude with geodesic extensions.

Next we introduce $\mathrm{CAT}(\kappa)$ spaces and move to a special class, CAT $(0)$ spaces, which is of most interest to us. We look at properties of these spaces and include a section on polarisation in $\mathrm{CAT}(0)$ spaces in which we introduce a polarisation identity for CAT(0) spaces and new work on angles. Next we look at 'building new spaces from old' including product spaces and using Reshetnyak's theorem.

Importantly for us, the nearest point projection algorithm works as expected in CAT(0) spaces. We then examine projections onto closed convex sets in CAT(0) spaces, which importantly prove to be nonexpansive. We conclude this section by examining analogues of weak convergence in CAT(0) spaces. Many Hilbert space arguments involving weak compactness can be replaced by asymptotic centre arguments or the notion of $\Delta$-convergence or $\phi$-convergence (which we take as the analogue of weak sequential convergence).

Thesis submitted to the University of Newcastle in July 2014; degree approved on 14 November 2014; supervisors Brailey Sims and George Willis.

(c) 2015 Australian Mathematical Publishing Association Inc. 0004-9727/2015 \$16.00 
After this, we begin our development of convex analysis in CAT(0) spaces. We introduce the concept of hyperplanes and half-spaces in CAT(0) and develop a separation theorem followed by a hyperplane characterisation of weak convergence in CAT(0) spaces. We move then to convex functions, tangent cones to points of a geodesic metric space, the differentiability of convex functions and finally subdifferentials.

We then examine fixed point theory in $\operatorname{CAT}(0)$ spaces and find that the notion of weak sequential convergence proves to be sufficient to develop a rich fixed point theory for nonexpansive-type mapping which parallels that in Hilbert space.

The final two chapters contain some of our major work in CAT(0) spaces. We prove that the projection algorithms commonly known as the 'project-project algorithm' and the 'project-project-average algorithm' in Hilbert space can be extended to CAT $(0)$ spaces. We also present an application of the alternating projection method to convex optimisation in CAT(0) spaces [1]. For the remainder of the thesis, we consider the reflection algorithm in $\mathrm{CAT}(0)$ spaces and find that while it is nonexpansive in spaces of constant curvature this is not generally true for spaces of nonconstant curvature. We develop a prototype CAT( 0 ) space of nonconstant curvature and use it both to show the failure of reflections to be nonexpansive and to investigate the Douglas-Rachford algorithm in such a space. We develop formulae for the geodesics in this space and use Maple to show that the Douglas-Rachford algorithm still appears to work in our prototype space, despite the lack of nonexpansivity.

\section{References}

[1] M. Bačák, I. Searston and B. Sims, 'Alternating projections in CAT(0) spaces', J. Math. Anal. Appl. 385 (2012), 599-607.

IAN SEARSTON, 160 Denton Park Drive, Aberglasslyn,

NSW 2320, Australia

e-mail: ianlynn@idl.net.au 